\title{
RMetS
}

Royal Meteorological Society

\section{A global shallow-water model on an icosahedral-hexagonal grid by a multi-moment constrained finite-volume scheme}

\author{
Chungang Chen, ${ }^{\text {a }}$ Juzhong Bin, ${ }^{\mathrm{b}}$ Feng Xiao, ${ }^{\mathrm{c}}$ Xingliang $\mathrm{Li}^{\mathrm{d}}$ and Xueshun Shen ${ }^{\mathrm{d}}$ \\ ${ }^{a}$ School of Human Settlement and Civil Engineering, Xi'an Jiaotong University, Xi'an, China \\ ${ }^{\mathrm{b}}$ LHD, Institute of Mechanics, Chinese Academy of Sciences, Beijing, China \\ ${ }^{c}$ Department of Energy Sciences, Tokyo Institute of Technology, Yokohama, Japan \\ ${ }^{\mathrm{d} C e n t e r ~ o f ~ N u m e r i c a l ~ W e a t h e r ~ P r e d i c a t i o n, ~ C h i n a ~ M e t e o r o l o g i c a l ~ A d m i n i s t r a t i o n, ~ B e i j i n g, ~ C h i n a ~}$ \\ ${ }^{\star}$ Correspondence to: C. G. Chen, 28 Xianning West Road, Xi’an, Shaanxi, 710049, China. \\ E-mail: cgchen@mail.xjtu.edu.cn
}

\begin{abstract}
A third-order global shallow-water model was developed on an icosahedral-hexagonal grid with the application of the multi-moment constrained finite-volume (MCV) method. Using the hexagonal grid, the sphere is divided into quasi-uniform elements and free of polar problems. The MCV model defines seven degrees of freedom (DOFs) as the prognostic variables which are the point values at the six vertices and the centre for each hexagonal element to construct a third-order scheme. The time evolution equations to update the DOFs are derived through the constraint conditions on different moments, i.e. the point value and the volume-integrated average (VIA) moments. Rigorous conservation is guaranteed by the constraint on the VIA through a flux form. The MCV formulation is very simple and easy to implement. We evaluated the model with benchmark tests, and the competitive results reveal the proposed model to be an accurate and practical framework for developing general circulation models.
\end{abstract}

Key Words: global model; icosahedral-hexagonal grid; multi-moment constraints; high-order scheme; shallow-water equations

Received 1 August 2012; Revised 29 December 2012; Accepted 18 March 2013; Published online in Wiley Online Library 22 May 2013

Citation: Chen CG, Bin JZ, Xiao F, Li XL, Shen XS. 2014. A global shallow-water model on an icosahedral-hexagonal grid by a multi-moment constrained finite-volume scheme. Q. J. R. Meteorol. Soc. 140: 639-650. DOI:10.1002/qj.2157

\section{Introduction}

High-quality quasi-uniform grids play an important role in improving the performance of general circulation models (GCMs). Being a quasi-uniform grid, the icosahedral geodesic grid was first proposed by Sadourny et al. (1968) and Williamson (1968) and projected an inscribed icosahedron onto the spherical surface to avoid the polar problems of the traditional latitude-longitude (lat-long) grid. In the past few decades, the icosahedral geodesic grid has been extensively used among the atmospheric and oceanic modelling community as one of the most preferred grids. Efforts have been made to develop global models using the icosahedral geodesic grid. Some representative studies include Lipscomb and Ringler et al. (2005), Miura (2007) and Skamarock and Menchaca (2010) for transport models, Tomita et al. (2001), Lee and MacDonald (2008), Ii and Xiao (2010) and Ringler et al. (2010) for shallow-water models and Majewski et al. (2002) and Satoh et al. (2008) for the non-hydrostatic dynamic core. A more complete survey can be found in the review paper by Staniforth and Thuburn (2012).

Both triangular and hexagonal elements can be generated from an icosahedron with quasi-uniform areas over the whole spherical surface which is highly desirable for global computations. Compared to the triangular Delaunay tessellation, the hexagonal Voronoi tessellation is more 
attractive in global grid uniformity, for the arrangement of local degrees of freedom (DOFs) for reconstruction, and for accuracy and robustness in flux computation. Weller et al. (2008) compared spherical shallow-water models using different grids, and demonstrated the favorable performance of the hexagonal grid.

One of the major drawbacks of an icosahedral grid is the unstructured connections among the mesh elements (St-Cyr et al., 2008; Ringler et al., 2008). As a result, researchers have met difficulties in developing high-order global models, which usually require considerably large stencils for spatial reconstructions. Moreover, selection and optimization of reconstruction stencils for high-order schemes on unstructured grids essentially affect the quality of the Vandermonde matrix, and are known to present a challenging task to the computational fluid dynamics (CFD) community. To our knowledge, most existing global models on an icosahedral grid possess second-order accuracy or less.

Another trend recently seen is the use of high-order methods with more local DOFs, such as the discontinuous Galerkin (DG) method (Cockburn and Shu, 1998) and the spectral element (SE) method (Patera, 1984), to construct more accurate dynamic cores on spherical grids. Some prototypes of this kind are found in Giraldo (2006), Nair et al. (2009), Dennis et al. (2005) and Dennis et al. (2012), among others. With more DOFs defined locally within each computational element, high-order spatial reconstructions can be built on a compact stencil (over a single cell in most cases). These high-order schemes are very beneficial for unstructured grids because they avoid spatial reconstructions over the large stencils. Nevertheless, applications of the DG and SE methods in global atmospheric and oceanic modelling, including the aforementioned studies, are mainly limited to the quadrilateral elements which are generated from either a cubed-sphere grid or a kite grid that first partitions an icosahedron into small triangles and then further divides the triangles into quadrilaterals (Staniforth and Thuburn, 2012). However, these high-order models are more computationally intensive compared to the conventional finite-volume method, and usually meet a more restrictive CFL condition for computational stability.

A more intuitive and efficient method, the so-called multimoment constrained finite volume (MCV) method, was proposed by Ii and Xiao (2009). As a high-order scheme with increased local DOFs, solution points are located within each mesh cell (control volume) where the point values (PVs) are updated as the prognostic variables. Given more local DOFs, cell-wise high-order reconstruction can be built. The point values are updated by time evolution equations which are derived from the constraint conditions on multimoments which are the PV and the volume-integrated average (VIA). The MCV method shows advantages in algorithmic simplicity, computational efficiency and a less restrictive CFL condition in comparison with other existing high-order schemes with local reconstructions. The MCV scheme is robust and well suited for constructing high-order models on unstructured grids (Akoh et al., 2010; Ii and Xiao, 2010). Ii and Xiao (2010) developed third- and fourth-order shallow-water models using the MCV scheme and triangular elements on an icosahedral grid.

In this article, instead of using triangular elements, we implement a MCV scheme for the shallow-water model on hexagonal mesh elements of an icosahedral grid. The hexagonal element, which has more boundary edges and larger angles between two intersecting edges, provides another more favorable mesh configuration to develop MCV high-order global models. Using the MCV scheme, we have already developed a global model on an icosahedral-hexagonal grid for scalar transport (Chen et al., 2012). By defining seven local DOFs over each hexagonal element, our model achieves third-order accuracy.

In this article, we extend our MCV model to the system of conservation laws and propose a global shallow-water model on an icosahedral-hexagonal grid. In our implementation, we define the water depth and momentum vector at the same location, which we have called the M-grid (described in Xiao et al., 2006). Since we always adopt at least two kinds of moments, the dispersion relation of the multi-moment scheme is different from the Arakawa A-grid. For example, the dispersion relation of a multi-moment model for the linearized shallow-water equations combines the advantages of both the A- and C-grids, and gives a reasonably accurate numerical dispersion for all deformation radii with respect to grid resolution. As other high-order schemes which use more than one DOF for local reconstructions, the multi-moment constraint scheme has computational modes. However, the computational modes are exponentially damped out due to the properly embedded numerical dissipation via the solutions of Riemann problems of the hyperbolic system.

The rest of this article is organized as follows. In section 2, the configuration of DOFs and the numerical formulations to discretise the shallow-water equations using the thirdorder MCV scheme on an icosahedral-hexagonal grid are described. Several benchmark tests for the shallow-water model on the sphere are computed and presented in section 3 to verify the convergence rate and performance of the present model. Finally, a short conclusion is given in section 4 to summarize this study.

\section{Global shallow-water model by a third- order MCV scheme}

\subsection{Governing equations}

To develop the global shallow-water model on the icosahedral-hexagonal grid, we adopted the governing equations in three-dimensional Cartesian coordinates (Williamson et al., 1992), instead of those in lat-long coordinates to get around the polar singularity. Governing equations of flux-form are written as

$$
\partial_{t} \mathbf{Q}+\partial_{x} \mathbf{E}+\partial_{y} \mathbf{F}+\partial_{z} \mathbf{G}=\mathbf{S},
$$

where dependent variables $\mathbf{Q}=[h, h u, h v, h w]^{\mathrm{T}}$ are water depth and momentum components in the $x, y$ and $z$ directions, and the flux functions are

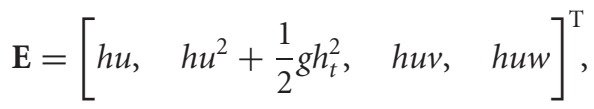

$$
\begin{aligned}
& \mathbf{F}=\left[\begin{array}{lll}
h v, & h u v, & h v^{2}+\frac{1}{2} g h_{t}^{2}, \quad h v w
\end{array}\right]^{\mathrm{T}} \\
& \mathbf{G}=\left[\begin{array}{llll}
h w, & h u w, & h v w, & h w^{2}+\frac{1}{2} g h_{t}^{2}
\end{array}\right]^{\mathrm{T}} .
\end{aligned}
$$


The source terms include Coriolis force and topographic effect as

$$
\mathbf{S}=\left[\begin{array}{c}
0 \\
\bar{R}(z h v-y h w)+g h_{s} \partial_{x} h_{t} \\
\bar{R}(x h w-z h u)+g h_{s} \partial_{y} h_{t} \\
\frac{f}{R}(y h u-x h v)+g h_{s} \partial_{z} h_{t}
\end{array}\right]
$$

where $(x, y, z)$ is the location in Cartesian coordinates, $h_{s}$ the height of bottom mountain, $h_{\mathrm{t}}=h+h_{\mathrm{s}}$ the total height of water surface and $f=2 \Omega z / R$ the Coriolis parameter. $R=$ $6371220 \mathrm{~m}, \Omega=7.292 \times 10^{-5} \mathrm{~s}^{-1}$ and $g=9.80616 \mathrm{~m} \mathrm{~s}^{-1}$ are the radius, angular velocity and gravity acceleration of the Earth, respectively.

Total height is adopted for the height gradient part of the flux terms (2)-(4). Solving governing equations of this form makes the numerical model automatically satisfy the 'C-property' (Xing and Shu et al., 2005), which means that an exact still solution should be always guaranteed when water is still and the total height is constant. Our previous study (Chen and Xiao, 2008) shows that a numerical scheme satisfying the 'C-property' can remove the non-physical oscillations in numerical solutions when topography is included.

Using the three-dimensional governing equations (1) to construct the shallow-water model on the sphere, which actually describes the two-dimensional flows in lat-long coordinates, requires a velocity (or momentum) correction to assure that the velocity (or momentum) vector is restricted in the tangential direction of the spherical surface. In this study, the predicted momentum vector $[h u, h v, h w]^{\mathrm{T}}$ at each Runge-Kutta substep is multiplied by the projection matrix (Williamson et al., 1992) as

$$
\mathcal{P}=\frac{1}{R^{2}}\left[\begin{array}{ccc}
R^{2}-x^{2} & -x y & -x z \\
-x y & R^{2}-y^{2} & -y z \\
-x z & -y z & R^{2}-z^{2}
\end{array}\right] .
$$

\subsection{Configuration of DOFs and spatial reconstruction}

The computational grid is generated as in our previous study (Chen et al., 2012). The spherical icosahedron is generated by projecting an inscribed icosahedron onto the sphere. The spherical triangular grid is then constructed on each piece of the spherical icosahedron by equidistantly dividing the edges of the icosahedron into $N$ arcs and $N$ is adopted to represent the grid resolution. The details of the above procedure can be found in the Appendix of Ii and Xiao (2010). Finally, the spherical hexagonal-pentagonal grid is obtained by simply connecting the centre of six or five (around twelve vertices of icosahedron) triangles sharing the same grid point.

Though no special technique is used in the present study to improve the quality of grid, our global MCV model gives uniform third-order convergence rate (shown in section 4 for benchmark tests), which reveals that the present model is robust for computational grids. This is one of the major merits of using 'localized' schemes on spherical hexagonal grids. Various techniques have been investigated to further improve the quality of the hexagonal grid, such as the strategy of using spring dynamics proposed by Tomita et al. (2001). These techniques can be easily transplanted into the present model and may well further improve the accuracy of the global model.
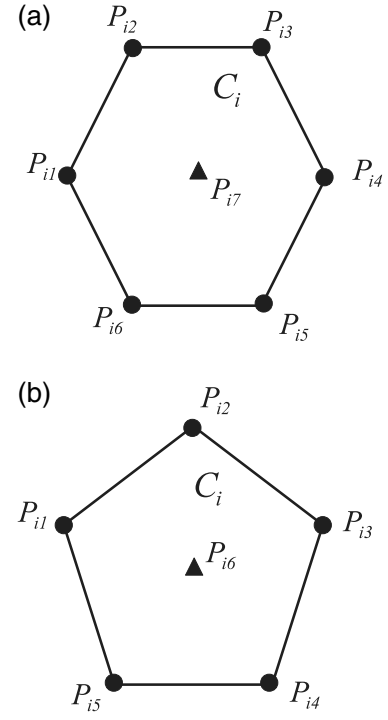

Figure 1. Configuration of DOFs for (a) hexagonal and (b) pentagonal elements.

Using the multi-moment concept, multiple DOFs within each element are defined and updated to build the local high-order spatial reconstruction. This has been proven in our previous work (Ii et al., 2005; Akoh et al., 2010; Ii and Xiao, 2010) to be a practical way to construct accurate, robust and efficient high-order schemes on unstructured grids. For the computational element $\mathcal{C}_{i}$, configuration of DOFs is shown in Figure 1. Within a hexagonal element (Figure 1(a)), seven local DOFs are defined as point values at six vertices ( $P_{i 1}$ to $P_{i 6}$ denoted by solid circles) and the centre of the element ( $P_{i 7}$ denoted by solid triangle). Similarly, within a pentagonal element (Figure 1(b)), six DOFs are defined including point-wise values at five vertices ( $P_{i 1}$ to $P_{i 5}$ denoted by solid circles) and the centre of the element ( $P_{i 6}$ denoted by solid triangle). The DOFs defined at vertices are shared by three adjacent elements. As a result, the total number of the DOFs adopted by the present model on grid $N$ is $30 N^{2}+2$, including $20 N^{2}$ DOFs at the vertices and $10 N^{2}+2$ at the cell centres.

Using DOFs within each element, a single-cell-based quadratic spatial reconstruction for any physical field $\psi$ can be obtained. For control volume $\mathcal{C}_{i}$, it is written as

$$
\begin{aligned}
\widehat{\psi}_{i}\left(\lambda^{(i)}, \theta^{(i)}\right)= & c_{00}+c_{10} \lambda^{(i)}+c_{01} \theta^{(i)} \\
& +c_{11} \lambda^{(i)} \theta^{(i)}+c_{20}\left(\lambda^{(i)}\right)^{2}+c_{02}\left(\theta^{(i)}\right)^{2} \\
& +c_{12}\left[\left(\lambda^{(i)}\right)^{2} \theta^{(i)}+\lambda^{(i)}\left(\theta^{(i)}\right)^{2}\right]
\end{aligned}
$$

for a hexagonal element and

$$
\begin{aligned}
\widehat{\psi}_{i}\left(\lambda^{(i)}, \theta^{(i)}\right)= & c_{00}+c_{10} \lambda^{(i)}+c_{01} \theta^{(i)} \\
& +c_{11} \lambda^{(i)} \theta^{(i)}+c_{20}\left(\lambda^{(i)}\right)^{2}+c_{02}\left(\theta^{(i)}\right)^{2}
\end{aligned}
$$

for a pentagonal element, where $\left(\lambda^{(i)}, \theta^{(i)}\right)$ is a rotated lat-long grid with origin at the centre of elements $\mathcal{C}_{i}$.

The coefficients are determined by solving the equation set provided by seven (hexagonal element) or six (pentagonal element) DOFs. The numerical procedure is the same as that adopted in the advection model (Chen et al., 2012). 


\subsection{Numerical formulations}

\subsubsection{Multi-moment constraints}

We define two kinds of moments (constraints) to derive the formulations for updating the DOFs defined above, i.e. the PV moment and VIA moment. They are defined for the physical field $\psi$ as follows.

- The PV moment is defined at the vertices of a control volume. At point $P_{i m}(m=1$ to $M-1)$ shown in Figure 1, it is written as

$$
\bar{P}_{i m}(t)=\psi\left(x_{i m}, y_{i m}, z_{i m}, t\right),
$$

where $M=7$ for a hexagonal element and $M=6$ for a pentagonal element.

- The VIA moment is defined over each control volume. For control volume $\mathcal{C}_{i}$, it is written as

$$
\bar{V}_{i}(t)=\frac{1}{\left|A_{i}\right|} \int_{\mathcal{C}_{i}} \psi(x, y, z, t) \mathrm{d} s,
$$

where $\left|A_{i}\right|$ is the area of control volume $\mathcal{C}_{i}$.

Shown above, we have $M$ local DOFs and can use spatial reconstruction (7) or (8) to connect the local DOFs and the multi-moment constraints by the following constraint relations:

$$
\psi_{i m}=\bar{P}_{i m}(m=1 \text { to } M-1)
$$

at the vertices and

$$
\psi_{i M}=\sum_{m=1}^{M-1}\left(\alpha_{i m} \bar{P}_{i m}\right)+\alpha_{i M} \bar{V}_{i}
$$

at the centre, where coefficients $\alpha_{i m}$ ( $m=1$ to $\left.M\right)$ are obtained by integrating the spatial reconstruction over the spherical hexagonal or pentagonal element. The quadrature formulations were given in Appendix B of Chen et al. (2012).

Equations (11) and (12) connect the unknown DOFs with the moments. So, once the time evolution equations for the moments PV and VIA are obtained, we can immediately get the time evolution equations to update the DOFs, which can be solved by a Runge-Kutta scheme. The time evolution equations in a semi-discretized form of PV and VIA moments are described as follows.

\subsubsection{Updating the PV moment}

Differential-form governing equations are adopted to update the PV moment. As shown in Figure 2, for example, the PV moment defined at point $P_{i 1}$ is updated using

$$
\partial_{t}\left(\bar{P}^{P} \mathbf{Q}_{i 1}\right)=-\left(\partial_{x} \mathbf{E}\right)_{i 1}-\left(\partial_{y} \mathbf{F}\right)_{i 1}-\left(\partial_{z} \mathbf{G}\right)_{i 1}+\mathbf{S}_{i 1} .
$$

The derivatives of the flux components, i.e. the derivatives of $\mathbf{E}, \mathbf{F}, \mathbf{G}$ with respect to $x, y$, and $z$ respectively, are computed at cell vertex $P_{i 1}$. The source term is calculated at $P_{i 1}$ using local values of dependent variables and coordinates for the Coriolis force, as well as the derivatives of total height with respect to $x, y$, and $z$ for the topographic term. The derivatives of total height are first evaluated within the

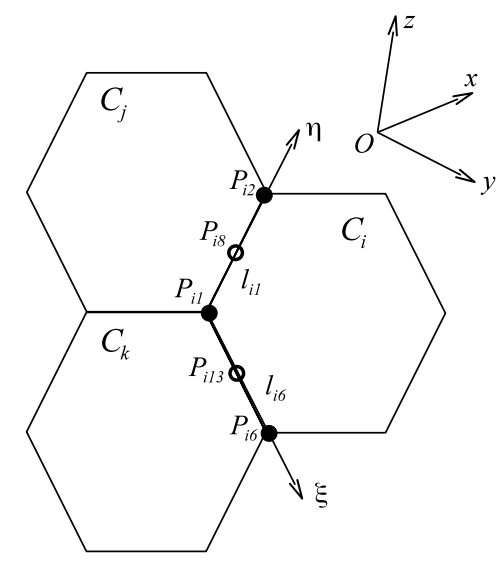

Figure 2. Derivative Riemann problem for updating the PV moment.

three elements sharing $P_{i 1}$ separately. Generally, these three values might be different, and an averaging is adopted to get derivatives in the topographic source term.

To construct the upwind scheme, a derivative (or general) Riemann problem is solved at $P_{i 1}$. For the sake of brevity, we only describe the discretization conducted in the $x$ direction. Formulations in the other two directions can be simply obtained by a similar procedure.

At point $P_{i 1}$, the derivative Riemann solver in the $x$ direction is written as (Ii and Xiao, 2009)

$$
\left(\partial_{x} \mathbf{E}\right)_{i 1}=\frac{1}{2}\left[\left(\partial_{x} \mathbf{E}\right)_{i 1}^{+}+\left(\partial_{x} \mathbf{E}\right)_{i 1}^{-}\right]+\mathbf{a}\left[\left(\partial_{x} \mathbf{E}\right)_{i 1}^{+}-\left(\partial_{x} \mathbf{E}\right)_{i 1}^{-}\right]
$$

where

$$
\mathbf{a}=\frac{1}{2} \mathbf{R} \operatorname{sgn}(\Lambda) \mathbf{R}^{-1}
$$

and matrixes $\Lambda$ and $\mathbf{R}$ consist of eigenvalues and eigenvectors of the Jacobian matrix $\mathcal{A}$ of flux component $\mathrm{E}$, i.e. $\mathcal{A}=\partial \mathbf{E} / \partial \mathbf{Q}$.

Since the point values of all the physical variables are available at the vertices where the DOFs are updated, the corresponding matrixes $\Lambda$ and $\mathbf{R}$ can be directly calculated.

The derivatives of flux component $\mathbf{E}$ with respect to $x$ are evaluated within upstream and downstream cells respectively, i.e. $\left(\partial_{x} \mathbf{E}\right)_{i 1}^{+}$and $\left(\partial_{x} \mathbf{E}\right)_{i 1}^{-}$in $(14)$. The formulations developed in Chen et al. (2012) for a global transport model on the same grid are used straightforwardly to evaluate these derivatives. As shown in Figure 2, we consider the calculation of $\left(\partial_{x} \mathbf{E}\right)_{i 1}^{-}$within the downstream cell in the $x$ direction, i.e. control volume $\mathcal{C}_{i}$. The numerical procedure is summarized as follows.

A local coordinate system $(\xi, \eta, r)$ is constructed along two sides $l_{i 6}(\xi)$ and $l_{i 1}(\eta)$, which intersect at point $P_{i 1}$, as well as the sphere radius $r$. The locations of points $P_{i 1}, P_{i 13}$, $P_{i 8}, P_{i 6}$ and $P_{i 2}$ are $(0,0, R),(0.5,0, R),(0,0.5, R),(1,0, R)$ and $(0,1, R)$ in the local coordinate system, where points $P_{i 8}$ and $P_{i 13}$ are the centres of the $\operatorname{arcs} l_{1}$ and $l_{6}$. Given the point-wise values of flux component $\mathbf{E}$ at these five points, we can easily calculate the derivatives of $\mathbf{E}$ with respect to $\xi$ and $\eta$ at point $P_{i 1}$ by

$$
\left.\begin{array}{l}
\left(\partial_{\xi} \mathbf{E}\right)_{i 1}=-3 \mathbf{E}_{i 1}+4 \mathbf{E}_{i 13}-\mathbf{E}_{i 6} \\
\left(\partial_{\eta} \mathbf{E}\right)_{i 1}=-3 \mathbf{E}_{i 1}+4 \mathbf{E}_{i 8}-\mathbf{E}_{i 2}
\end{array}\right\}
$$

Using the chain rule, we can get $\left(\partial_{x} \mathbf{E}\right)_{i 1}^{-}$. The details can be found in (32) and (33) of Chen et al. (2012). 
At three vertices of $\mathcal{C}_{i}, \mathbf{E}_{i 1}, \mathbf{E}_{i 2}$ and $\mathbf{E}_{i 6}$ are calculated using DOFs defined there directly. In order to determine $\mathbf{E}_{i 8}$ and $\mathbf{E}_{i 13}$, the point-wise values of dependent variables at the centre of these two sides, i.e. $\mathbf{Q}_{i 8}$ and $\mathbf{Q}_{i 13}$, are evaluated as follows.

We first evaluate them within two adjacent cells respectively as

$$
\begin{array}{ll}
\mathbf{Q}_{i 8}^{(i)}=\widehat{\mathbf{Q}}_{i}\left(\lambda_{i 8}^{(i)}, \theta_{i 8}^{(i)}\right), & \mathbf{Q}_{i 8}^{(j)}=\widehat{\mathbf{Q}}_{j}\left(\lambda_{i 8}^{(j)}, \theta_{i 8}^{(j)}\right), \\
\text { and } \\
\mathbf{Q}_{i 13}^{(i)}=\widehat{\mathbf{Q}}_{i}\left(\lambda_{i 13}^{(i)}, \theta_{i 13}^{(i)}\right), & \mathbf{Q}_{i 13}^{(k)}=\widehat{\mathbf{Q}}_{k}\left(\lambda_{i 13}^{(k)}, \theta_{i 13}^{(k)}\right),
\end{array}
$$

where $\widehat{\mathbf{Q}}_{i}\left(\lambda^{(i)}, \theta^{(i)}\right), \widehat{\mathbf{Q}}_{j}\left(\lambda^{(j)}, \theta^{(j)}\right)$ and $\widehat{\mathbf{Q}}_{k}\left(\lambda^{(k)}, \theta^{(k)}\right)$ denote the cell-wise interpolation functions (7) or (8) over cell elements $\mathcal{C}_{i}, \mathcal{C}_{j}$ and $\mathcal{C}_{k}$ respectively.

In general, $\mathbf{Q}_{i 8}^{(i)} \neq \mathbf{Q}_{i 8}^{(j)}$ and $\mathbf{Q}_{i 13}^{(i)} \neq \mathbf{Q}_{i 13}^{(k)}$. The numerical flux $\mathbf{E}$ at these points is then computed by averaged values of dependent variables, i.e. $\mathbf{Q}_{i 8}=\left(\mathbf{Q}_{i 8}^{(i)}+\mathbf{Q}_{i 8}^{(j)}\right) / 2$ and $\mathbf{Q}_{i 13}=\left(\mathbf{Q}_{i 13}^{(i)}+\mathbf{Q}_{i 13}^{(k)}\right) / 2$.

\subsubsection{Updating the VIA moment}

As in our previous studies, VIA constraints are updated through the flux-form formulation to guarantee mass conservation in the numerical model. For any dependent variable $Q^{(\beta)}$ ( $\beta=1$ to 4 , denoting the water height and the components of momentum in three directions), the governing equation in flux-form for the VIA moment over the control volume $\mathcal{C}_{i}$, i.e. ${ }^{\bar{V} Q^{(\beta)}}{ }_{i}$, is written as

$$
\partial_{t}\left(\overline{{ }^{V} Q^{(\beta)}}{ }_{i}\right)=-\frac{1}{\left|A_{i}\right|} \int_{\mathcal{C}_{i}}\left(\nabla \cdot \mathbf{H}^{(\beta)}\right) \mathrm{d} s+\frac{1}{\left|A_{i}\right|} \int_{\mathcal{C}_{i}} S^{(\beta)} \mathrm{d} s,
$$

where $\mathbf{H}^{(\beta)}=\left(E^{(\beta)}, F^{(\beta)}, G^{(\beta)}\right)$ denotes the flux vector.

The second term on the right-hand side of (19) is calculated through seven- or six-point quadrature within a hexagonal or pentagonal element, respectively. The coefficients are found in (12).

The first term on the right-hand side of (19) is the surface integral of divergence of the flux vector over the spherical element. Using Gauss's theorem, it is rewritten as

$$
\begin{aligned}
\int_{\mathcal{C}_{i}}\left(\nabla \cdot \mathbf{H}^{(\beta)}\right) \mathrm{d} s= & \sum_{m=1}^{M-1} \int_{l_{i m}}\left(\mathbf{H}^{(\beta)} \cdot \mathbf{n}_{l_{i m}}\right) \mathrm{d} l \\
& +\frac{2}{R} \int_{\mathcal{C}_{i}}\left(\mathbf{H}^{(\beta)} \cdot \mathbf{n}_{\mathcal{C}_{i}}\right) \mathrm{d} s
\end{aligned}
$$

where $\mathbf{n}_{l_{i m}}$ is the outward unit normal direction of side $l_{i m}$ (tangent to the spherical surface), $R$ is radius of the sphere and $\mathbf{n}_{\mathcal{C}_{i}}$ is the outward unit normal of spherical element $\mathcal{C}_{i}$.

The first term on the right-hand side of (20) is the line integral along the edges of the control volume. It is calculated through a three-point Simpson's rule. For example, we consider the flux computation along the side $l_{i 1}$ of control volume $\mathcal{C}_{i}$ as shown in Figure 3 ,

$$
\int_{l_{i 1}}\left(\mathbf{H}^{(\beta)} \cdot \mathbf{n}_{l_{i 1}}\right) \mathrm{d} l=\frac{\left|l_{i 1}\right|}{6}\left(\mathbf{H}_{i 1}^{(\beta)}+4 \mathbf{H}_{i 8}^{(\beta)}+\mathbf{H}_{i 2}^{(\beta)}\right) \cdot \mathbf{n}_{l_{i 1}},
$$

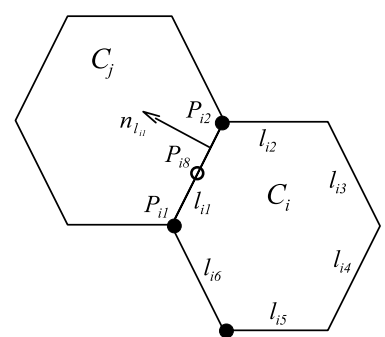

Figure 3. Evaluation of numerical flux across the cell's boundary.

where $\left|l_{i 1}\right|$ is the length of $\operatorname{arc} l_{i 1}$.

As we have discussed above, point values at two vertices $\mathbf{H}_{i 1}^{(\beta)}$ and $\mathbf{H}_{i 2}^{(\beta)}$ are calculated by DOFs defined there directly. Here we determine $\mathbf{H}_{i 8}^{(\beta)}$ by solving a Riemann problem at the centre of the arc $l_{i 1}$, which is shared by control volumes $\mathcal{C}_{i}$ and $\mathcal{C}_{j}$. The point-wise value of the flux vector in the normal direction is computed by $\mathbf{F}^{n}=n_{l_{i 1}}^{x} \mathbf{E}+n_{l_{i 1}}^{y} \mathbf{F}+n_{l_{i 1}}^{z} \mathbf{G}$, and $n_{l_{i 1}}^{x}, n_{l_{i 1}}^{y}$ and $n_{l_{i 1}}^{z}$ are components of the unit outward normal vector $\mathbf{n}_{l_{i 1}}$ in different directions.

At the centre of each side, recall that we have obtained two different values for the dependent variables from the spatial reconstructions of two adjacent cells, i.e. $\mathbf{Q}_{i 8}^{(i)}$ and $\mathbf{Q}_{i 8}^{(j)}$ at point $P_{i 8}$. First, two discontinuous values of the flux vector are obtained at this point as

$$
\mathbf{F}_{i 8}^{n+}=\mathbf{F}^{n}\left(\mathbf{Q}_{i 8}^{(i)}\right) \quad \text { and } \quad \mathbf{F}_{i 8}^{n-}=\mathbf{F}^{n}\left(\mathbf{Q}_{i 8}^{(j)}\right) \text {, }
$$

where superscript ' + ' denotes the upstream cell $\mathcal{C}_{i}$ according to the outward normal direction $\mathbf{n}_{l_{i 1}}$ and '-' denotes the downstream cell $\mathcal{C}_{j}$.

Second, we solve the Riemann problem in the normal direction to determine the numerical flux at the centre of edge $l_{i 1}$. We get

$$
\mathbf{F}_{i 8}^{n}=\frac{1}{2}\left(\mathbf{F}_{i 8}^{n+}+\mathbf{F}_{i 8}^{n-}\right)+\mathbf{a}^{n}\left(\mathbf{F}_{i 8}^{n+}-\mathbf{F}_{i 8}^{n-}\right),
$$

where

$$
\mathbf{a}^{n}=\frac{1}{2} \mathbf{R}^{n} \operatorname{sgn}\left(\Lambda^{n}\right)\left(\mathbf{R}^{n}\right)^{-1}
$$

$\Lambda^{n}$ and $\mathbf{R}^{n}$ are the diagonal matrix of the eigenvalues and the matrix of corresponding eigenvectors of the Jacobian matrix $\mathcal{A}^{n}=\partial \mathbf{F}^{n} / \partial \mathbf{Q}$, which are evaluated through averaging $\mathbf{Q}_{i 8}=\left(\mathbf{Q}_{i 8}^{(i)}+\mathbf{Q}_{i 8}^{(j)}\right) / 2$.

Finally, at point $P_{i 8}$ we get the flux vector as

$$
\mathbf{H}_{i 8}^{(\beta)} \cdot \mathbf{n}_{l_{i 1}}=F_{i 8}^{n(\beta)} .
$$

The second term on the right-hand side of (20) is a surface integral over the spherical cell for a correction due to the fact that the flux vector $\mathbf{H}^{(\beta)}(\beta=2$ to 4$)$ is not tangential to the spherical surface. Considering the expressions of flux components shown in (2), (3), (4), this term is written for different variables separately as

$$
\int_{\mathcal{C}_{i}}\left(\mathbf{H}^{(1)} \cdot \mathbf{n}_{\mathcal{C}_{i}}\right) \mathrm{d} s=0
$$


Table 1. Normalized errors and convergence rate of case 2 at day 5 on refined grids with $\gamma=0$.

\begin{tabular}{|c|c|c|c|c|c|c|}
\hline \multirow[t]{2}{*}{ Grid } & \multicolumn{2}{|c|}{$l_{1}$} & \multicolumn{2}{|c|}{$l_{2}$} & \multicolumn{2}{|c|}{$l_{\infty}$} \\
\hline & error & order & error & order & error & order \\
\hline$N=9$ & $6.633 e-4$ & - & $7.172 e-4$ & - & $1.023 e-3$ & - \\
\hline$N=18$ & $8.272 \mathrm{e}-5$ & 3.00 & $9.008 e-5$ & 2.99 & $1.276 \mathrm{e}-4$ & 3.00 \\
\hline$N=36$ & $1.034 \mathrm{e}-5$ & 3.00 & $1.128 \mathrm{e}-5$ & 3.00 & $1.597 \mathrm{e}-5$ & 2.99 \\
\hline$N=72$ & $1.293 \mathrm{e}-6$ & 3.00 & $1.410 \mathrm{e}-6$ & 3.00 & $1.997 \mathrm{e}-6$ & 3.00 \\
\hline
\end{tabular}

for the continuity equation and

$$
\begin{aligned}
\int_{\mathcal{C}_{i}}\left(\mathbf{H}^{(2)} \cdot \mathbf{n}_{\mathcal{C}_{i}}\right) \mathrm{d} s & =\frac{1}{2} g \int_{\mathcal{C}_{i}}\left(n_{\mathcal{C}_{i}}^{x} h_{t}^{2}\right) \mathrm{d} s, \\
\int_{\mathcal{C}_{i}}\left(\mathbf{H}^{(3)} \cdot \mathbf{n}_{\mathcal{C}_{i}}\right) \mathrm{d} s & =\frac{1}{2} g \int_{\mathcal{C}_{i}}\left(n_{\mathcal{C}_{i}}^{y} h_{t}^{2}\right) \mathrm{d} s, \\
\int_{\mathcal{C}_{i}}\left(\mathbf{H}^{(4)} \cdot \mathbf{n}_{\mathcal{C}_{i}}\right) \mathrm{d} s & =\frac{1}{2} g \int_{\mathcal{C}_{i}}\left(n_{\mathcal{C}_{i}}^{z} h_{t}^{2}\right) \mathrm{d} s
\end{aligned}
$$

for the momentum equations in different directions, where $n_{\mathcal{C}_{i}}^{x}, n_{\mathcal{C}_{i}}^{y}$ and $n_{\mathcal{C}_{i}}^{z}$ are the $x, y$ and $z$ components of unit vector $\mathbf{n}_{\mathcal{C}_{i}}$, the surface integral is accomplished by 7-point (within hexagonal cell) or 6-point (within pentagonal cell) quadrature formulation and the coefficients are derived from (12) as the second term on the right-hand side of (19).

After the discretization of spatial differential terms, we obtain a set of semi-discretised equations, and a Runge-Kutta scheme for ordinary differential equation is then used for time updating. To achieve third-order accuracy, the third-order TVD Runge-Kutta scheme (Shu, 1988 ) is adopted. The numerical procedure is same as the time integration in our transport model (Chen et al., 2012).

We have presented in this section the third-order MCV model for global shallow-water equations on the icosahedral-hexagonal grid. Using local DOFs, this model builds high-order reconstructions over a single-cell stencil and is very suited for global models on an icosahedral spherical grid. Implementing the MCV scheme on a hexagonal tessellation results in a concise and easy-to-use discretization formulation which has rigorous numerical conservation and third-order accuracy, as will be shown next.

\section{Numerical tests}

\subsection{Williamson's test case 2: Steady-state geostrophic flow}

The height field is written in lat-long coordinates as

$$
g h=g h_{0}-\frac{u_{0}}{2}\left(2 R \Omega+u_{0}\right)(\sin \theta \cos \gamma-\cos \lambda \cos \theta \sin \gamma)^{2},
$$

where $u_{0}=2 \pi R /(12$ days $), g h_{0}=2.94 \times 10^{4} \mathrm{~m}^{2} / \mathrm{s}^{2}$, and $\gamma$ represents the angle between rotation axis and the Earth's axis.

The velocity field $\left(u_{\lambda}, u_{\theta}\right)$ in lat-long coordinates is specified as

$$
\left.\begin{array}{l}
u_{\lambda}=u_{0}(\cos \theta \cos \gamma+\sin \theta \cos \lambda \sin \gamma), \\
u_{\theta}=-u_{0} \sin \lambda \sin \gamma
\end{array}\right\}
$$

The initial conditions generate a balanced geostrophic flow, so the height and velocity fields should keep their
Table 2. Normalized errors of case 2 at day 5 on grid $N=18$ in different rotation directions.

\begin{tabular}{llll}
\hline Direction & $l_{1}$ error & $l_{2}$ error & $l_{\infty}$ error \\
\hline$\gamma=0$ & $8.272 \mathrm{e}-5$ & $9.008 \mathrm{e}-5$ & $1.276 \mathrm{e}-4$ \\
$\gamma=\pi / 4$ & $7.155 \mathrm{e}-5$ & $8.222 \mathrm{e}-5$ & $1.778 \mathrm{e}-4$ \\
$\gamma=\pi / 2$ & $7.988 \mathrm{e}-5$ & $9.052 \mathrm{e}-5$ & $9.052 \mathrm{e}-5$ \\
\hline
\end{tabular}

initial distribution during computation. Since the height field is considerably smooth and the exact solution is known to be same as the initial condition, we run this test on a series of refined grids to numerically check third-order accuracy of the proposed shallow-water model.

We integrate for 5 days on grids of different refining levels and show in Table 1 the normalized errors $l_{1}, l_{2}$ and $l_{\infty}$ (defined following Williamson et al., 1992) of the height field for eastward rotation. Uniformly third-order accuracy is verified in this test. Our MCV model achieves third-order accuracy for the system of conservation laws, as did our previous study for scalar advection (Chen et al., 2012). In other rotation directions, the same convergence rates are observed and are not given for the sake of brevity. We show the normalized errors for tests on grid $N=18$ with different rotation directions in Table 2 . The $l_{1}$ and $l_{2}$ errors are almost independent of the rotation direction. $l_{\infty}$ error shows a slightly larger value for the $\gamma=\pi / 4$ case. This is probably because the flow path in this case contains more pentagonal cells.

This test case was also reported by Tomita et al. (2001) and Ringler et al. (2010) on an icosahedral-hexagonal grid. At day 5, our results (Table 1 ) are better than those of Tomita et al. (2001) (their Figure 10), where their grids of level 4, 5, 6, 7 adopt 2562, 10242,40 962, 163842 DOFs, a little more than our grids $N=9, N=18, N=36$ and $N=72$ with 2 432, 9722,38 882, 155522 DOFs, respectively. In Ringler et al. (2010), this was integrated to day 12 and numerical errors are shown in their Figure 7. Normalized errors of the present model on refined grids are similarly depicted in Figure 4. Although our model gives larger errors on the coarsest grid, it converges much faster with refining resolutions and the present MCV model is more accurate on fine grids. Compared with the MCV model on an icosahedral-triangular grid (Ii and Xiao, 2010), a more uniform convergence rate is observed in the present study.

Figure 5 in Ringler et al. (2010) shows the normalized $l_{2}$ and $l_{\infty}$ errors after integrating case 2 for one year by the TRiSK* ${ }^{*}$ model. Since the TRiSK model pays particular attention to the balance and conservation, the numerical result arrived at a balanced state and errors did not increase

* so called after Thuburn et al. (2009). 


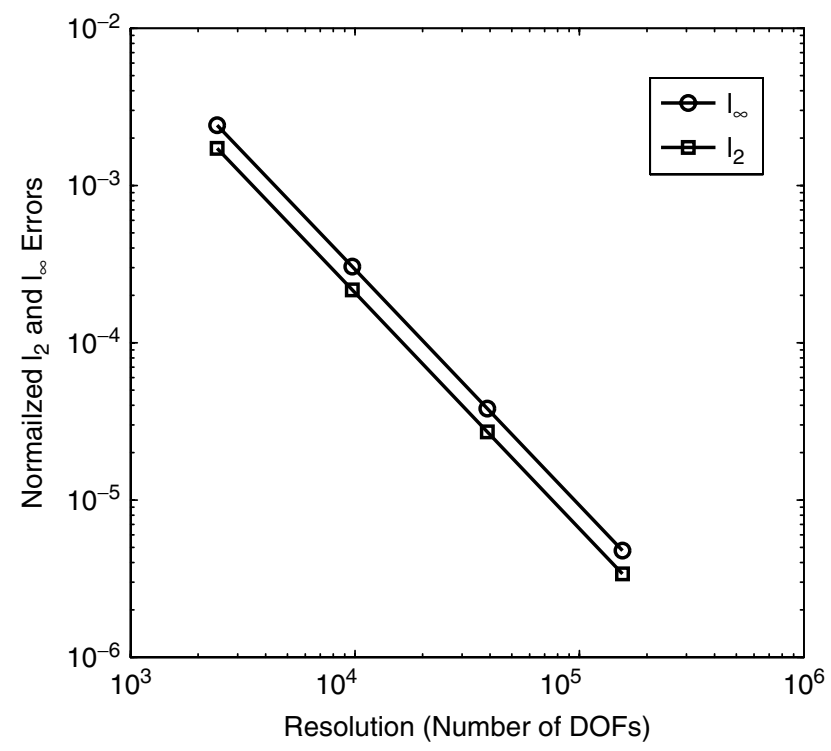

Figure 4. Convergence of the height field of case 2 after integrating for 12 days on refined grids.

with time in their result for case 2. Figure 5 gives the normalized $l_{2}$ and $l_{\infty}$ errors after integrating case 2 for one year by the present model. The present model has numerical dissipation and therefore does not conserve the total energy. As a result, the two normalized errors linearly increase with time during the integration.

The numerical solution and absolute error of the height field for eastward rotation on grid $N=9$ after integrating the MCV model for 5 days are shown in Figure 6. The distribution of absolute error shows a five-wave pattern due to the patch boundaries on the spherical icosahedron. To compare with the multi-moment model on the cubedsphere grid shown in Figure 10 in Chen and Xiao (2008), we depict the numerical result on grid $N=72$ for northeast rotation in Figure 7. The normalized errors on grid $N=72$ by the present model have the similar magnitude (of order $10^{-6}$ ) as those shown in Figure 10 in Chen and Xiao (2008) on the cubed-sphere grid. However, much larger noise is found around the patch boundaries on the cubed sphere. This explains why we obtain a very uniform convergence rate by the present model. It can be expected that we can obtain a more uniform convergence rate on the icosahedral grid than the cubed-sphere grid with high resolutions because the excessive errors generated by patch boundaries usually

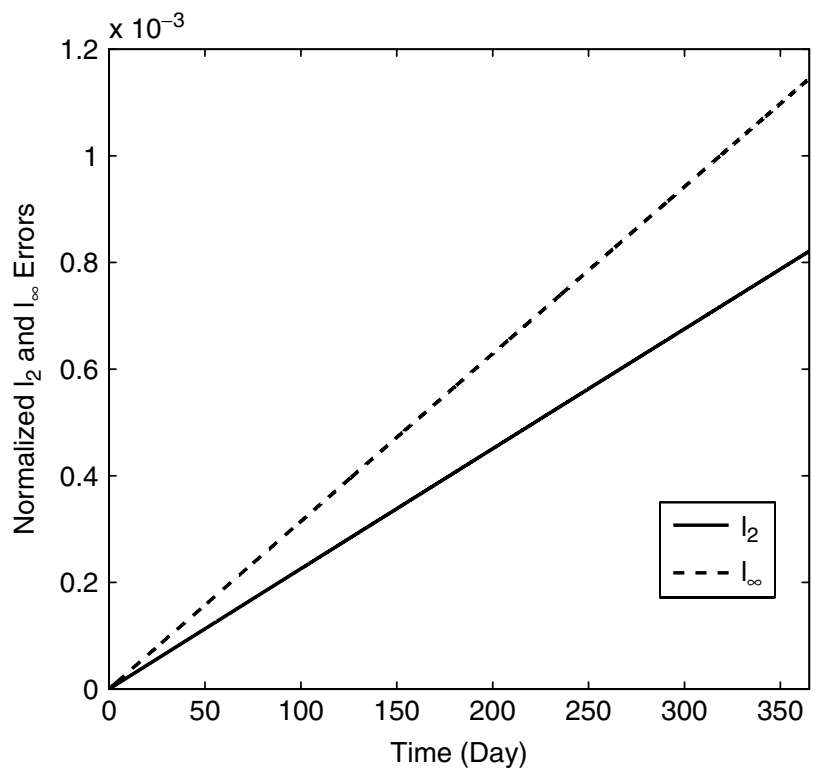

Figure 5. Normalized $l_{2}$ and $l_{\infty}$ errors of the height field of case 2 after integrating for one year on grid $N=36$.

converge more slowly than the truncation errors of the spatial discretizations within each patch.

\subsection{Williamson's test case 5: Zonal flow over an isolated mountain}

Balanced height and velocity fields are given as in the previous test, except the computational parameters are chosen as $h_{0}=5960 \mathrm{~m}$ and $u_{0}=20 \mathrm{~m} \mathrm{~s}^{-1}$. Additionally, a bottom mountain centred at $\left(\lambda_{c}, \theta_{c}\right)=(3 \pi / 2, \pi / 6)$ is specified as

$$
h_{\mathrm{s}}=h_{\mathrm{s} 0}\left(1-\frac{d}{d_{0}}\right)
$$

where $\quad d=\min \left[d_{0}, \sqrt{\left(\lambda-\lambda_{\mathrm{c}}\right)^{2}+\left(\theta-\theta_{\mathrm{c}}\right)^{2}}\right]$ and parameters are chosen as $h_{\mathrm{s} 0}=2000 \mathrm{~m}, d_{0}=\pi / 9$.

This test checks the performance of numerical models dealing with the topographic source term. The 'C-property' mentioned in section 2 is of essential importance in this test. Non-physical oscillations can be found along the mountain boundary in numerical solutions not satisfying the 'C-property', for example in the high-resolution spectral (a)

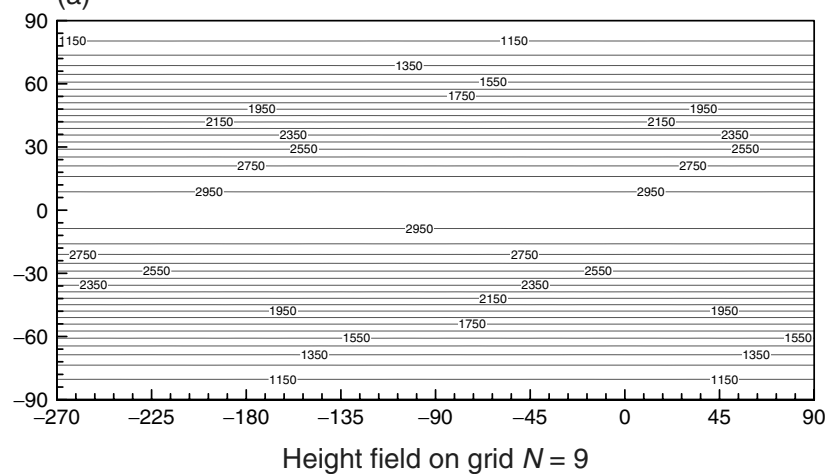

(b)

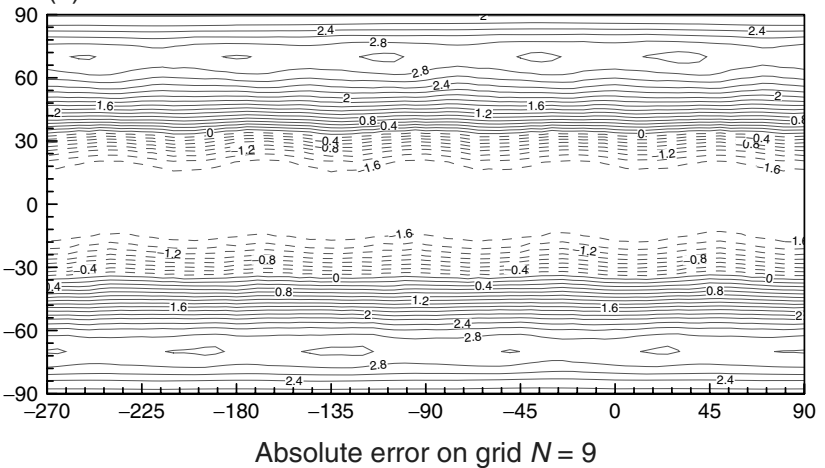

Figure 6. (a) Height field solution and (b) absolute error of the water depth in Williamson's standard test case 2 with $\gamma=0$ on grid $N=9$ (No. of DOFs: 2432) at day 5. 
(a)

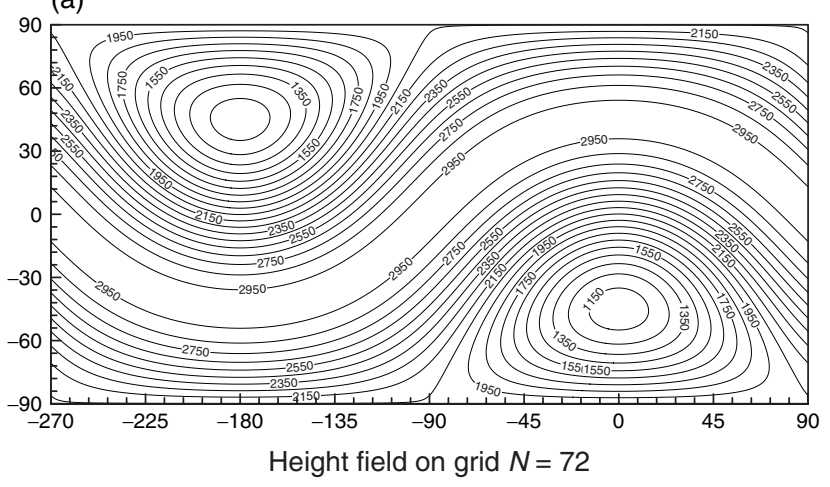

(b)

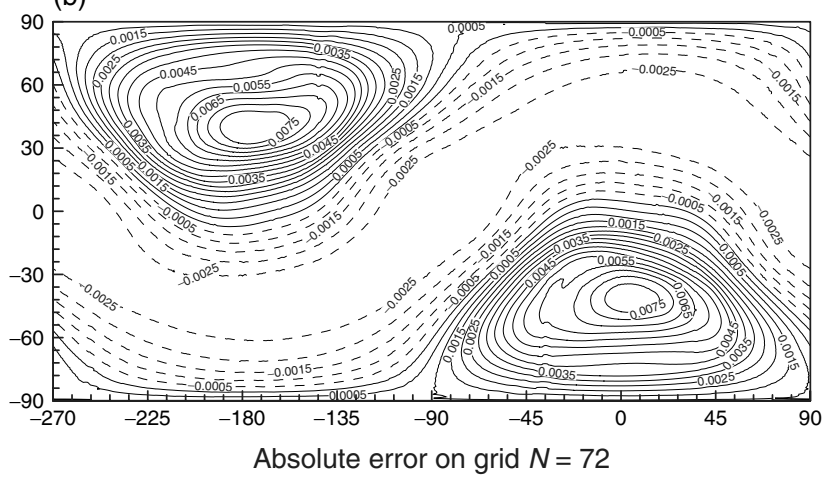

Figure 7. As Figure 6, but for the test with $\gamma=\pi / 4$ on grid $N=72$ (No. of DOFs: 155 522).

(a)

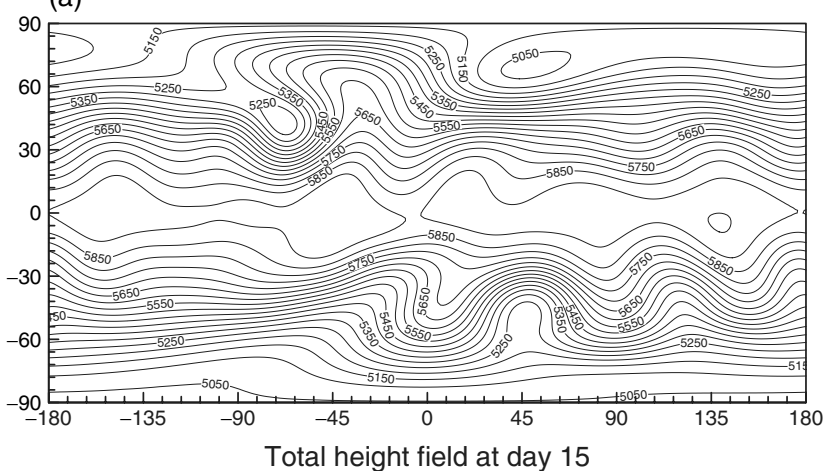

(b)

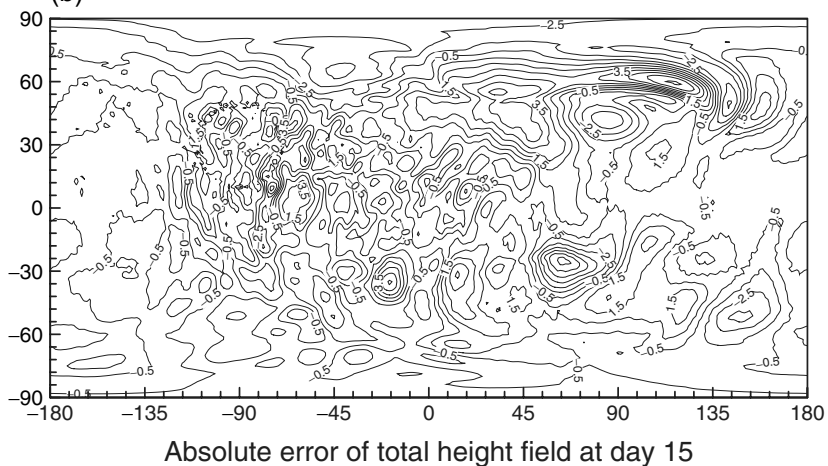

Figure 8. Numerical solutions of the (a) total height field and (b) absolute error of Williamson's standard test case 5 on grid $N=36$ (No. of DOFs: 38882 ) at day 15.

transform solution on grid T213 shown in Jakob-Chien et al. (1995) (their Figure 5.1).

Numerical results of the total height field after integrating the proposed MCV model for 15 days on grid $N=36$ are shown in Figure 8(a). The present model rigorously guarantees the 'C-property', thus there is not any visible oscillation around the bottom mountain. There is no analytic solution for this test. The absolute error of the total height field is calculated against the spectral transform solution to this test on T426 grid (available online at http://icon.enes.org/; provided by the German National Meteorological Service, DWD) and shown in Figure 8(b). It is observed that the significance errors are generated around the topography in the spectral transform solutions on T42 and T63 grids (Figure 5.2 in Jakob et al., 1993), while it is not a significant problem to the present model.

The present model conserves the total mass to machine precision. Additionally, the conservation errors of the total energy and potential enstrophy are small due to the accuracy of the MCV model. The normalized conservation errors of the total energy and potential enstrophy are shown in Figure 9 for simulations on grids $N=9, N=18, N=36$ and $N=72$. Unlike Jakob-Chien et al. (1995), we give the absolute values of errors for clear illustration on a (a)

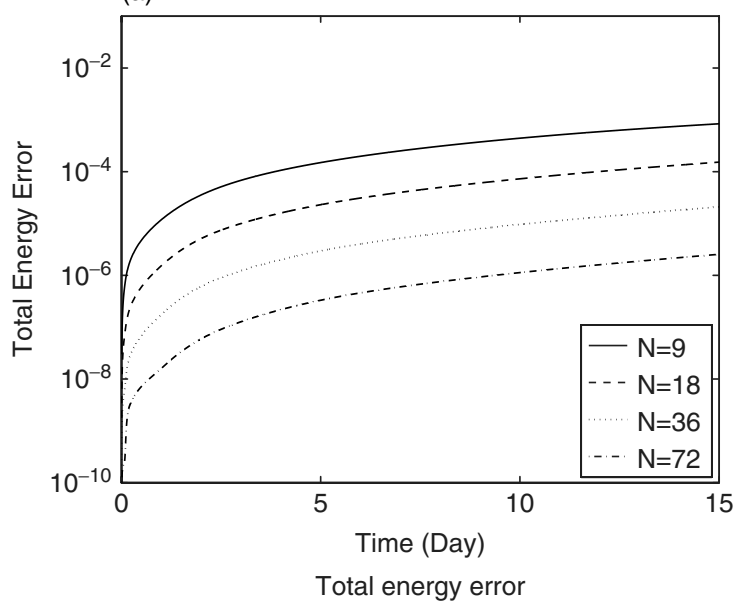

(b)

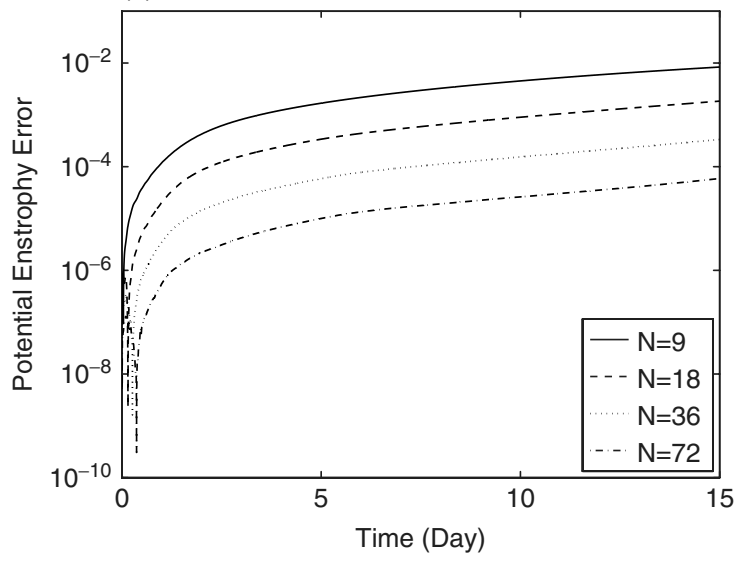

Potential enstrophy error

Figure 9. Time history of normalized (a) total energy error and (b) potential enstrophy error of Williamson's standard test case 5 on refined grids. 
(a)

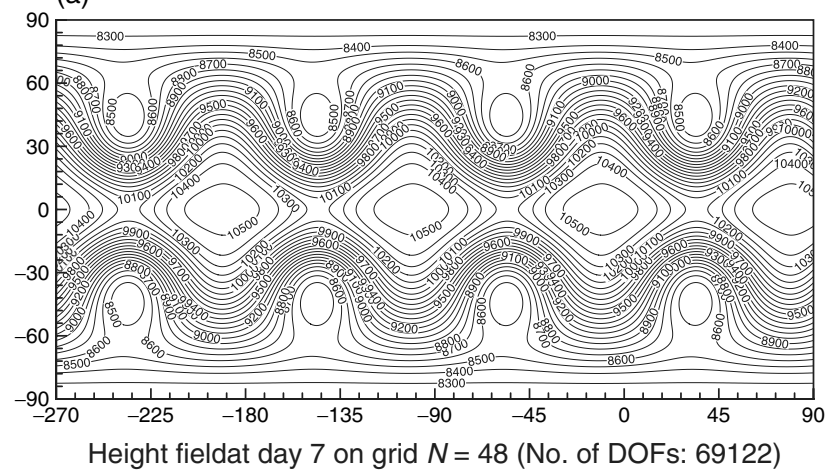

(c)

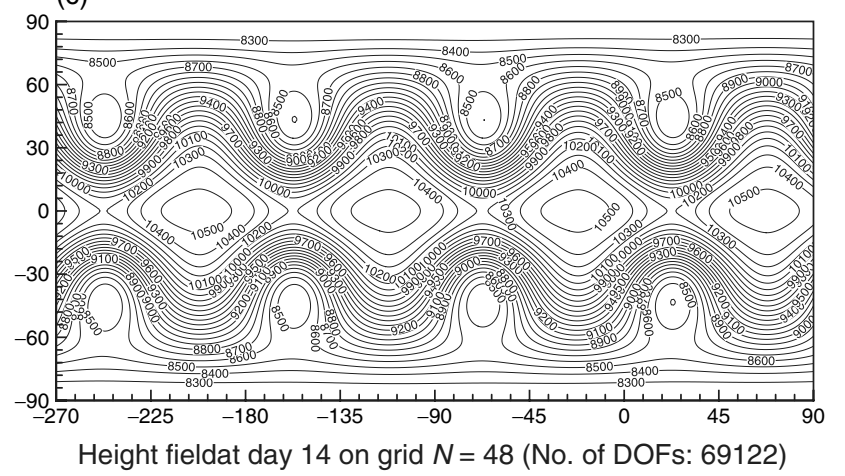

(b)

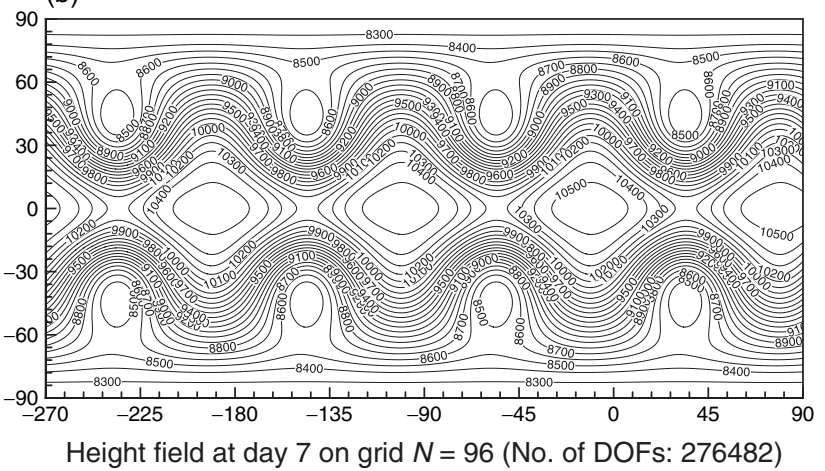

(d)

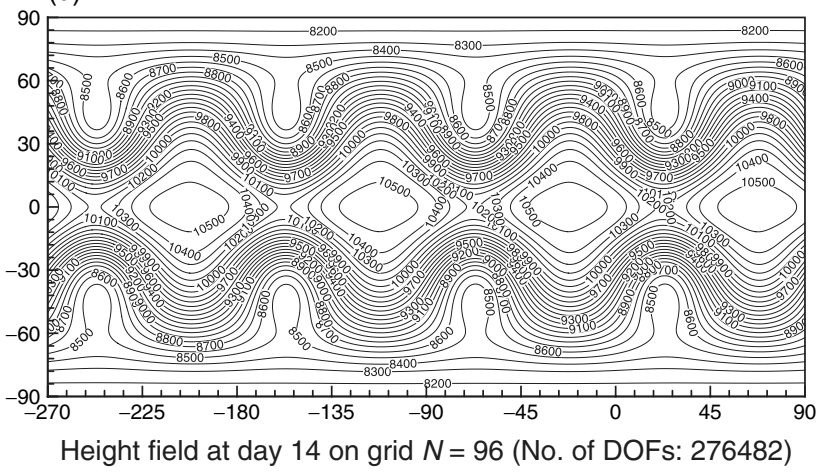

Figure 10. Numerical solutions of Williamson's standard test case 6 on different grids: height field at day 7 on grid (a) $N=48$ with 69122 DOFs, and (b) $N=96$ with 276482 DOFs. (c, d) are as (a,b), but at day 14 .

(a)

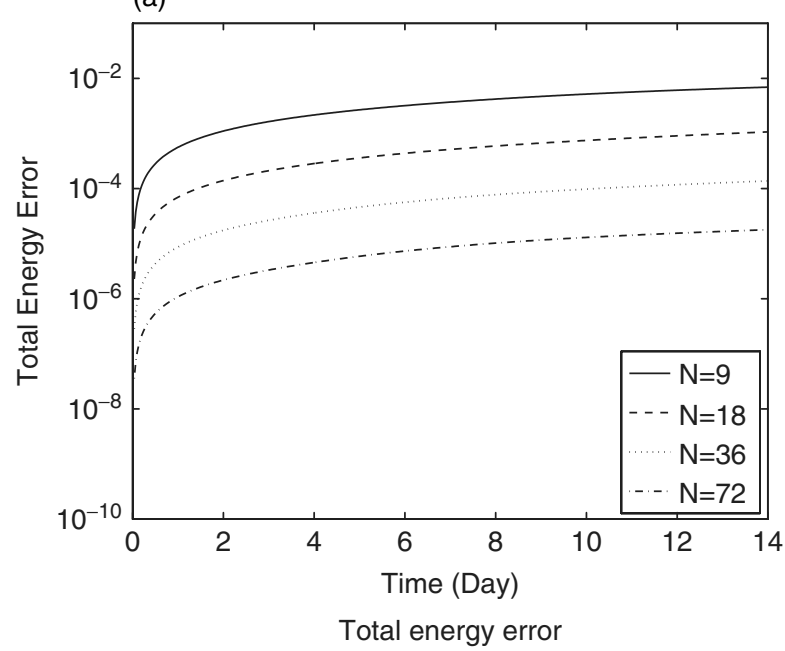

(b)

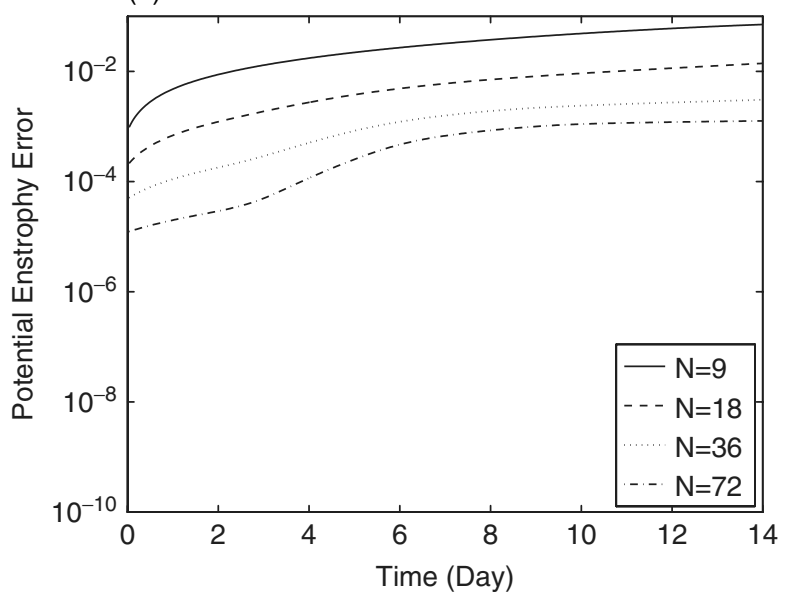

Potential enstrophy error

Figure 11. As Figure 9, but for Williamson's standard test case 6 on refined grids.

logarithmic scale. With refined grid resolution, conservation errors continue to decrease. The conservation error of the total energy by our model on grid $N=36$ is competitive with the spectral transform solution on grid T42 (Figure 5.3 in Jakob-Chien et al., 1995).

\subsection{Williamson's test case 6: Rossby-Haurwitz wave}

The Rossby-Haurwitz wave still provides a good test bed for global middle-term simulations. The details of the set-up of this test case were given in Williamson et al. (1992). The flow field of the Rossby-Haurwitz wave test is complicated and consists of phenomena of different scales. A high-order model with less numerical dissipation is more suitable to simulate this test. Similar to the isolated mountain test, there is no existing analytic solution. We consider the spectral transform solution on the fine T213 grid (Jakob-Chien et al., 1995) as the reference for its high-order accuracy.

Numerical solutions of the present model on different grids with $N=48$ and $N=96$ are shown in Figure 10 for the height field at day 7 and day 14 . Overall, both the basic structure and phase speed of our results agree well with the reference solution at day 7 and day 14. Comparing the numerical results with different grid resolutions, structures look almost identical at day 7. At day 14, obvious difference between numerical results with different grid resolutions is observed at the 8500 contour. It is closed on coarse 


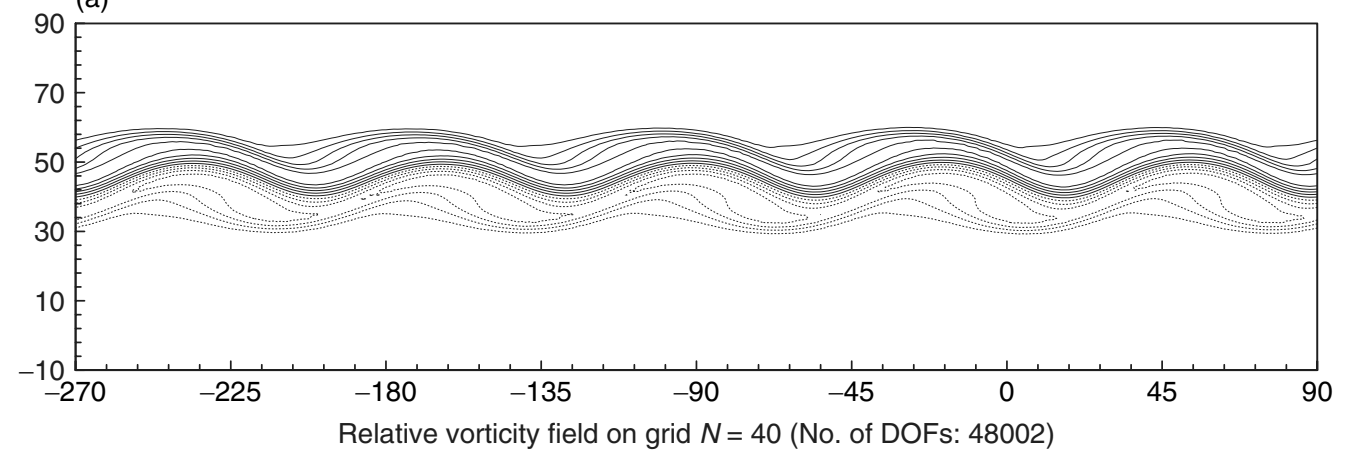

(b)

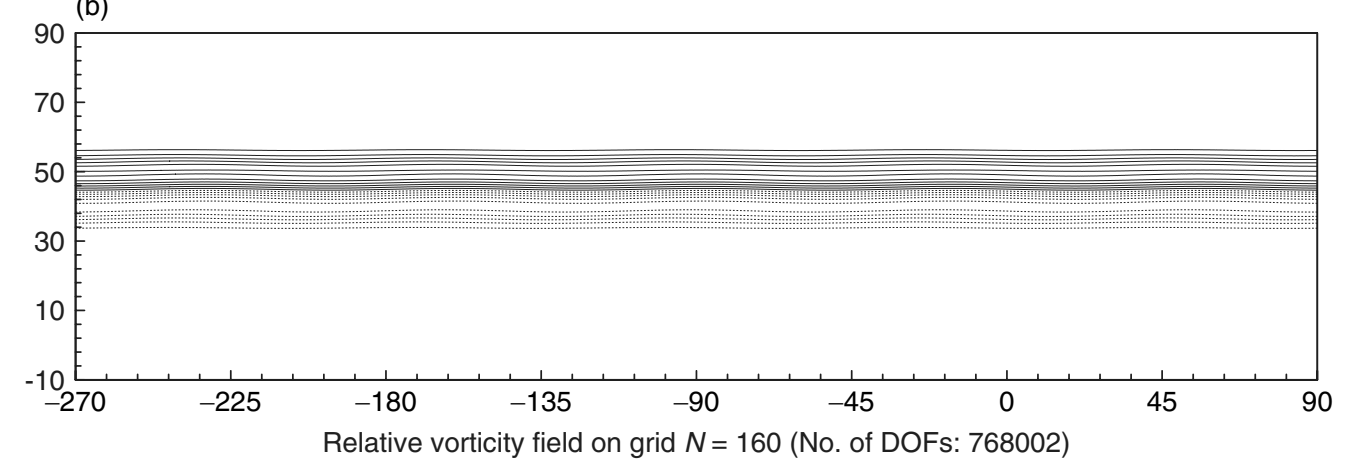

Figure 12. Numerical solutions of the relative vorticity field of the balanced jet flow at day 5 on (a) grid $N=40$ with 48002 DOFs, and (b) grid $N=160$ with 768002 DOFs.

grid $N=48$. Furthermore, the 10500 circle is found to be larger on fine grid $N=96$. Similar changes are found in the spectral transform solutions on T42 and T63 grids (Figure 5.7 in Jakob-Chien et al., 1995). The main reason for this change is the reduced numerical dissipation due to the smaller grid spacing.

Mass is exactly conserved by the present model. Normalized conservation errors of the total energy and potential enstrophy are shown in Figure 11 for simulations on grids $N=9, N=18, N=36$ and $N=72$. As in the previous test, conservation errors continue to decrease with the increase of grid resolution. Our solution on grid $N=36$ is competitive with the spectral transform solution on grid T42 in terms of total energy error (Figure 5.9 in Jakob-Chien et al., 1995).

\subsection{Perturbed jet flow}

A perturbed jet flow test was proposed by Galewsky et al. (2004). A balanced height and velocity field are given similarly as Williamson's test case 2 , but within a very narrow belt zone (Galewsky et al., 2004, give details of the set-up). As a result, the balance relation in the solution is easily destroyed by numerical errors including the initial imbalance and the truncation errors. We first test the balanced set-up without any perturbation in the height field. Numerical results of the relative vorticity field at day 5 are shown in Figure 12 for simulations on grids $N=40$ and $N=160$. On coarse grid $N=40$, the 5 -wave phenomenon is visible mainly due to the extra numerical errors caused by five internal boundaries between different patches of the spherical icosahedron. On the fine grid $N=160$, the balance condition is preserved during numerical simulation. Additionally, we give evolution of normalized $l_{1}$ errors of the height field in Figure 13 for simulations on refined

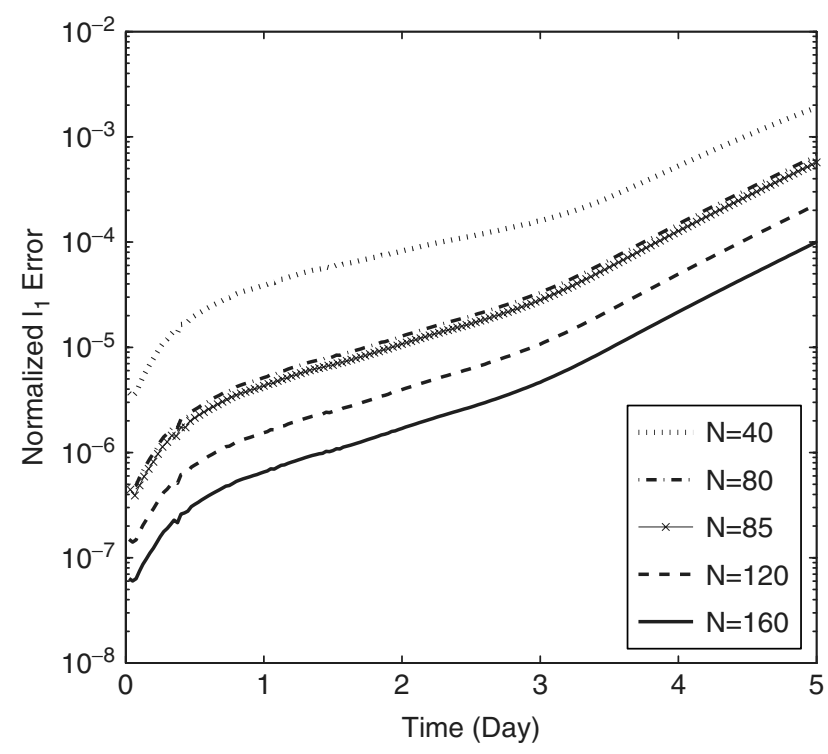

Figure 13. Normalized $l_{1}$ errors of height field of the balanced jet flow computed on refined grids.

grids. The number of DOFs on grid $N=85$ is equivalent to those of the tests shown in Figure 7 of Weller (2012). The normalized $l_{1}$ errors by three different models (the multimoment model on triangular mesh, the multi-moment on hexagonal mesh and the TRiSK model) at day 5 are of similar magnitude. The errors of two multi-moment models have the same evolution pattern, i.e. the error grows constantly during 5 days. Whereas, in the TRiSK model, numerical error grows very slowly in the first two to three days, as shown in Figure 7(a) in Weller (2012), and then grows more rapidly than two multi-moment models. 

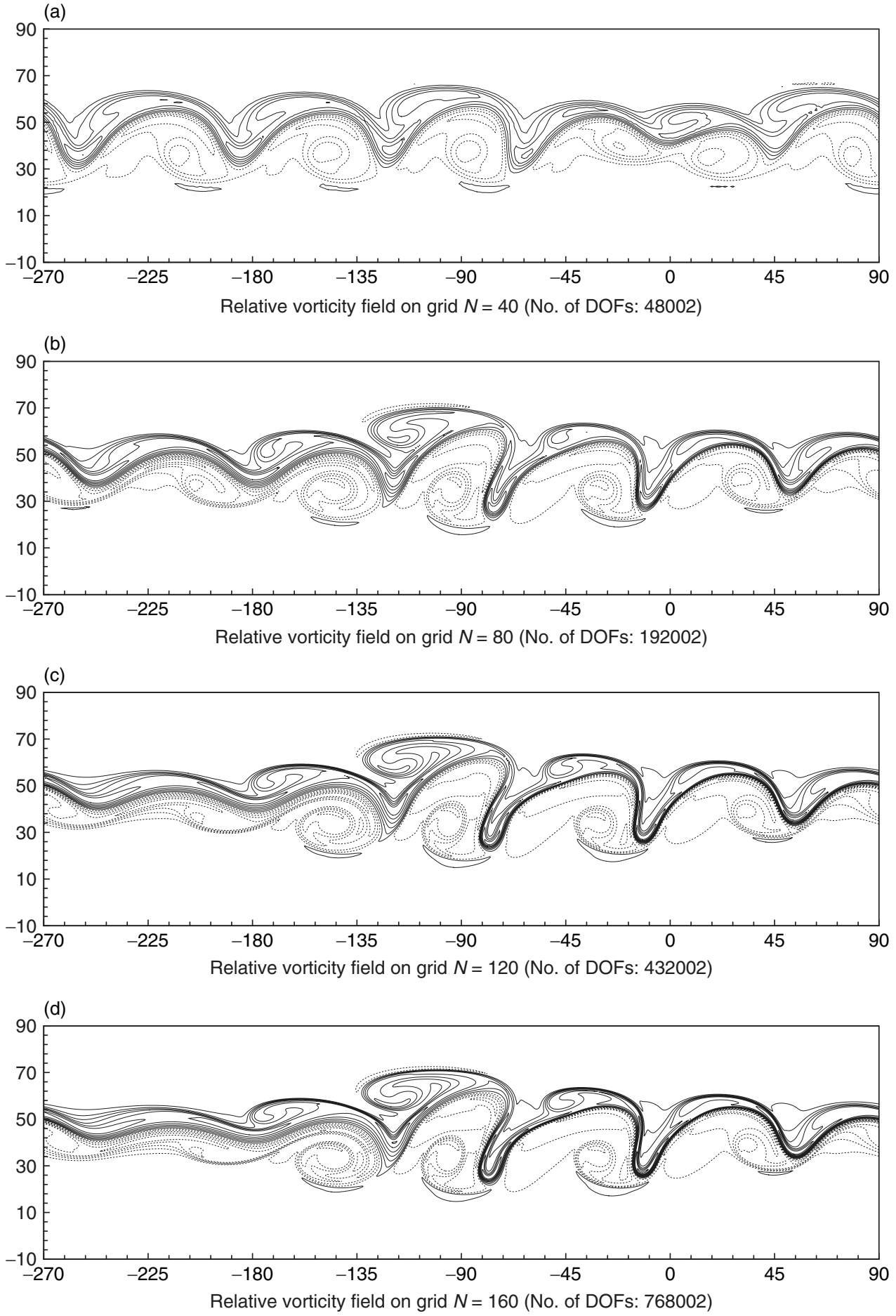

Figure 14. Numerical solutions of relative vorticity field of the perturbed jet flow at day 6 on various refined grids: (a) $N=40$ with 48002 DOFs, (b) $N=80$ with 192002 DOFs, (c) $N=120$ with 432002 DOFs, and (d) $N=160$ with 768002 DOFs.

Second, we add a small perturbation to the height field to test the perturbed jet flow. This test case runs on a series of refined grids to check the convergence of relative vorticity fields at day 6 . The reference solution is given in Galewsky et al. (2004) using a spectral transform model on fine grid T341. Numerical results of the relative vorticity field of the perturbed jet flow by integrating the MCV model for 6 days are shown in Figure 14 for simulations on grids $N=40, N=80, N=120$ and $N=160$. On the coarse grid with $N=40$, the result (Figure 14(a)) looks very different from the reference solution and the wave pattern is dominated by numerical errors. On the grid with
$N=80$ (Figure 14(b)), the difference between the numerical solution and the reference one is apparent only in the area from longitude $-270^{\circ}$ to $-180^{\circ}$. On the grids with $N=120$ and $N=160$, the numerical results (Figure 14(c,d)) look almost the same and the flow structures agree well with the reference solution.

\section{Conclusion}

We have extended our global transport model (Chen et al., 2012) to a system of conservation laws (shallowwater equations) by implementing the MCV method on 
an icosahedral-hexagonal spherical grid. All computational variables (unknowns) are the point values of physical fields defined at the vertices and the centre of elements; this allows us to construct a third-order spatial discretization scheme based on a single-cell stencil. The time evolution equations of both PVs and VIAs are used as the constraint conditions to derive the equations for updating the unknowns. The VIA constraint leads to a finite-volume formulation, and ensures rigorous numerical conservation. The numerical formulation of the MCV method on a hexagonal element is simple and easy to use.

A third-order accuracy was proved in our previous study (Chen et al., 2012) for the advection equation by Taylor expansion analysis and numerical experiments. In this article we have verified its third-order accuracy for a nonlinear system of conservation laws. Widely used benchmark tests were simulated by the present model and the numerical results are competitive with most existing advanced models on an icosahedral grid. Furthermore, the MCV scheme also shows advantage in computational efficiency compared with other local high-order schemes due to, for example, a larger available CFL number (Ii and Xiao, 2009). The proposed high-order scheme on an icosahedral-hexagonal grid is a very promising framework for developing general circulation models.

\section{Acknowledgements}

This study was supported by National Key Technology R\&D Program of China (project number 2012BAC22B01), Natural Science Foundation of China (project number 10902116) and JSPS KAKENHI (grant number 24560187).

\section{References}

Akoh R, Ii S, Xiao F. 2010. A multi-moment finite-volume formulation for shallow-water equations on unstructured mesh. J. Comput. Phys. 229: 4567-4590.

Chen CG, Xiao F. 2008. Shallow-water model on cubed sphere by multimoment finite-volume method. J. Comput. Phys. 227: 5019-5044.

Chen CG, Bin JZ, Xiao F. 2012. Global multimoment constrained finitevolume scheme for advection transport on the hexagonal geodesic grid. Mon. Weather Rev. 140: 941-955.

Cockburn B, Shu C-W. 1998. TVB RungeKutta local projection discontinuous Galerkin finite-element method for conservation laws. V: multidimensional systems. J. Comput. Phys. 141: 199-224.

Dennis JM, Fournier A, Spotz WF, St-Cyr A, Taylor MA, Thomas SJ, Tufo H. 2005. High-resolution mesh convergence properties and parallel efficiency of a spectral element atmospheric dynamical core. Internat. J. High Perform. Comput. Appl. 19: 225-235.

Dennis JM, Edwards J, Evans KJ, Guba O, Lauritzen PH, Mirin AA, St-Cyr A, Taylor MA, Worley PH. 2012. CAM-SE: A scalable spectral element dynamical core for the Community Atmosphere Model. Internat. J. High Perform. Comput. Appl. 26: 74-89.

Galewsky J, Scott K, Polvani LM. 2004. An initial-value problem for testing numerical models of the global shallow-water equations. Tellus A 56: 429-440.

Giraldo FX. 2006. High-order triangle-based discontinuous Galerkin methods for hyperbolic equations on a rotating sphere. J. Comput. Phys. 214: 447-465.

Ii S, Xiao F. 2009. High-order multi-moment constrained finite-volume method. Part I: Basic formulation. J. Comput. Phys. 228: 3669-3707.

Ii S, Xiao F. 2010. A global shallow-water model using high-order multi-moment constrained finite-volume method and icosahedral grid. J. Comput. Phys. 229: 1774-1796.

Ii S, Shimuta M, Xiao F. 2005. A fourth-order and single-cell-based advection scheme on unstructured grids using multi-moments. Comput. Phys. Comm. 173: 17-33.

Jakob R, Hack JJ, Willamson DL. 1993. 'Solutions to the shallow-water test set using the spectral transform method'. Report NCAR/TN$388+$ STR. NCAR: Boulder, CO.

Jakob-Chien R, Hack JJ, Williamson DL. 1995. Spectral transform solutions to the shallow-water test. J. Comput. Phys. 119: 164-187.

Lee JL, MacDonald AE. 2008. A finite-volume icosahedral shallow-water model on a local coordinate. Mon. Weather Rev. 137: 1422-1437.

Lipscomb WH, Ringler T. 2005. An incremental remapping transport scheme on a spherical geodesic grid. Mon. Weather Rev. 133: 2235-2250.

Liu Y, Vinokur M, Wang ZJ. 2006. Spectral difference method for unstructured grids. I: Basic formulation. J. Comput. Phys. 216: $780-801$.

Majewski D, Liermann D, Prohl P, Ritter B, Buchhold M Hanisch T, Paul G, Wergen W. 2002. The operational global icosahedral-hexagonal grid-point model GME: description and highresolution tests. Mon. Weather Rev. 130: 319-338.

Miura H. 2007. An upwind-biased conservative advection scheme for spherical hexagonal-pentagonal grids. Mon. Weather Rev. 135: 4038-4044.

Nair RD, Choi H-W, Tufo HM. 2009. Computational aspects of a scalable high-order discontinuous Galerkin atmospheric dynamical core. Comput. Fluids 38: 309-319.

Patera AT. 1984. A spectral element methd for fluid dynamics: Laminar flow in a channel expansion. J. Comput. Phys. 54: 468-488.

Ringler T, Ju LL, Gunzburger M. 2008. A multi-resolution method for climate system modeling: application of spherical centroidal Voronoi tessellations. Ocean Dyn. 58: 475-498.

Ringler T, Thuburn J, Klemp JB, Skamarock WC. 2010. A unified approach to energy conservation and potential vorticity dynamics on arbitrarily structured C-grids. J. Comput. Phys. 229: 3065-3090.

Sadourny R, Arakawa A, Mintz Y. 1968. Integration of the nondivergent barotropic vorticity equation with an icosahedral-hexagonal grid for the sphere. Mon. Weather Rev. 96: 351-356.

Satoh M, Matsuno T, Tomita H, Miura H, Nasuno T, Iga S. 2008. Non-hydrostatic icosahedral atmospheric model (NICAM) for global cloud-resolving simulations. J. Comput. Phys. 227: 3486-3514.

Shu CW. 1988. Total variation diminishing time discretization. SIAM J. Sci. Statist. Comput. 9: 1073-1084.

Skamarock WC, Menchaca M. 2010. Conservative transport schemes for spherical geodesic grids: High-order reconstructions for forward-intime schemes. Mon. Weather Rev. 138: 4497-4508.

St-Cyr S, Jablonowski C, Dennis JM, Tufo HM, Thomas SI. 2008. A comparison of two shallow-water models with non-conforming adaptive grids. Mon. Weather Rev. 136: 1898-1922.

Staniforth A, Thuburn J. 2012. Horizontal grids for global weather and climate prediction models: a review. Q. J. R. Meteorol. Soc. 138: 1-26.

Thuburn J, Ringler T, Skamarock WC, Klemp JB. 2009. Numerical representation of geostrophic modes on arbitrarily structured C-grids. J. Comput. Phys. 228: 8321-8335.

Tomita H, Tshugawa M, Satoh M, Goto K. 2001. Shallow-water model on a modified icosahedral geodesic grid by using spring dynamics. J. Comput. Phys. 174: 579-613.

Wang ZJ. 2002. Spectral (finite) volume method for conservation laws on unstructured grids: basic formulation. J. Comput. Phys. 178: 210-251.

Weller H. 2012. Controlling the computational modes of the arbitrarily structured C-grid. Mon. Weather Rev. 140: 3220-3234.

Weller H, Weller HG, Fournier A. 2008. Voronoi, Delaunay, and block-structured mesh refinement for solution of the shallow-water equations on the sphere. Mon. Weather Rev. 137: 4208-4224.

Williamson DL. 1968. Integration of the barotropic vorticity equation on a spherical geodesic grid. Tellus 20: 642-653.

Williamson DL, Drake JB, Hack JJ, Jakob R, Swarztrauber PN. 1992. A standard test set for numerical approximations to the shallow-water equations in spherical geometry. J. Comput. Phys. 102: 211-224.

Xiao F, Peng XD, Shen XS. 2006. A finite-volume grid using multimoment geostrophic adjustment. Mon. Weather Rev. 134: 2515-2526.

Xing Y, Shu CW. 2005. High-order finite-difference WENO schemes with the exact conservation property for the shallow-water equations. J. Comput. Phys. 208: 206-227. 